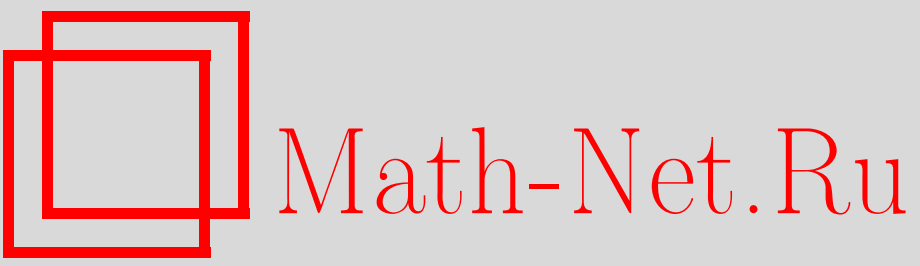

С. М. Гусейн-Заде, Ф. Дельгадо, А. Кампильо, Интегрирование по эйлеровой характеристике по пространству функций и полином Александера особенности плоской кривой, УМН, 2000, том 55, выпуск 6, 127-128

DOI: https://doi.org/10.4213/rm338

Использование Общероссийского математического портала Math-Net.Ru подразумевает, что вы прочитали и согласны с пользовательским соглашением

http: //www.mathnet.ru/rus/agreement

Параметры загрузки:

IP : 34.239 .49 .27

26 апреля 2023 г., $17: 08: 14$ 


\title{
ИНТЕГРИРОВАНИЕ ПО ЭЙЛЕРОВОЙ ХАРАКТЕРИСТИКЕ ПО ПРОСТРАНСТВУ ФУНКЦИЙ И ПОЛИНОМ АЛЕКСАНДЕРА ОСОБЕННОСТИ ПЛОСКОЙ КРИВОЙ
}

\author{
С. М. ГУСЕЙН-ЗАДЕ, Ф. ДЕЛЬГАДО, А. КАМПИЛЬО
}

Пусть $\Delta^{C}\left(t_{1}, \ldots, t_{r}\right)$ - полином Александера приведенной особенности плоской кривой $C=$ $\bigcup_{i=1}^{r} C_{i}\left(C_{i}\right.$-еенеприводимые компоненты), т.е. зацепления $C \cap S_{\varepsilon}^{3} \subset S_{\varepsilon}^{3}$ для достаточно малого $\varepsilon>0$ (см., например, [1]). Вообще говоря, полином Александера определен только с точностью до умножения на моном $\pm t_{1}^{n_{1}} \cdots t_{r}^{n_{r}}$. Мы филксируем его, полагая, что он действительно является полиномом (т.е. не содержит переменных в отрицательных степенях) и что $\Delta^{C}(0, \ldots, 0)=1$. Пусть $\zeta_{C}(t)$ - дзета-функция преобразования классической монодромии особенности $C$, т.е. ростка функции $f:\left(\mathbb{C}^{2}, 0\right) \rightarrow(\mathbb{C}, 0)$ такого, что $C=\{f=0\}$ (см., например, [2]). Для $r>1$ имеем $\zeta_{C}(t)=\Delta^{C}(t, \ldots, t)\left(\right.$ для $\left.r=1 \zeta_{C}(t)=\Delta^{C}(t) /(1-t)\right)$.

В [3] было показано, что все коэффициенты полинома Александера $\Delta^{C}(\underline{t})\left(\underline{t}=\left(t_{1}, \ldots, t_{r}\right)\right)$ могут быть выражены как эйлеровы характеристики некоторых явным образом описанных пространств - дополнений к системам проективных гиперплоскостей в проективных пространствах. Для особенности гиперповерхности произвольной размерности числа Лефшеца итераций преобразования классической монодромии были описаны как эйлеровы характеристики некоторых подпространств в пространстве (усеченных) дуг [4]. Этот результат связан с теорией интегрирования по отношению к эйлеровой характеристике в пространстве дуг. Здесь мы обсуждаем аналогичное понятие (интегрирование по отношению к эйлеровой характеристике) в проективизации $\mathbb{P} \mathscr{O}_{\mathbb{C}^{n}, 0}$ кольца $\mathscr{O}_{\mathbb{C}^{n}, 0}$ ростков функций на пространстве $\mathbb{C}^{n}$ в начале координат (рассматриваемом здесь как линейное пространство) и показываем, что полином Александера и дзета-функция особенности плоской кривой могут быть выражены в виде некоторых интегралов по пространству $\mathbb{P} \mathscr{O}_{\mathbb{C}^{2}, 0}$ по отношению к эйлеровой характеристике.

Пусть $J_{\mathbb{C}^{n}, 0}^{k}$-пространство $k$-струй функций в начале координат в пространстве $\mathbb{C}^{n}\left(J_{\mathbb{C}^{n}, 0}^{k}=\right.$ $\mathscr{O}_{\mathbb{C}^{n}, 0} / \mathfrak{m}^{k+1} \cong \mathbb{C}\left(\begin{array}{c}n+k \\ k\end{array}\right)$, где $\mathfrak{m}$-максимальньй идеал в кольце $\left.\mathscr{O}_{\mathbb{C}^{n}, 0}\right)$. Для комплексного линейного пространства $L$ (конечно- или бесконечномерного) обозначим через $\mathbb{P} L$ его проективизацию $(L \backslash\{0\}) / \mathbb{C}^{*}$, пусть $\mathbb{P}^{*} L$ - несвязное объединение $\mathbb{P} L$ и точки (в некотором смысле $\left.\mathbb{P}^{*} L=L / \mathbb{C}^{*}\right)$. Имеются естественные отображения $\pi_{k}: \mathbb{P}_{\mathbb{C}^{n}, 0} \rightarrow \mathbb{P}^{*} J_{\mathbb{C}^{n}, 0}^{k}, \pi_{k, \ell}: \mathbb{P}^{*} J_{\mathbb{C}^{n}, 0}^{k} \rightarrow \mathbb{P}^{*} J_{\mathbb{C}^{n}, 0}^{\ell}$ для $k \geqslant \ell$. Над $\mathbb{P} J_{\mathbb{C}^{n}, 0}^{\ell} \subset \mathbb{P}^{*} J_{\mathbb{C}^{n}, 0}^{\ell}$ отображение $\pi_{k, \ell}$ является локально тривиальным расслоением, слоем которого является комплексное линейное пространство размерности $C_{n+k}^{k}-C_{n+\ell}^{\ell}$.

ОПредЕЛЕниЕ. Говорят, что подмножество $X \subset \mathbb{P}_{\mathbb{C}^{n}, 0}$ является цилиндрическим, если $X=\pi_{k}^{-1}(Y)$ для некоторого полуалгебраического подмножества $Y \subset \mathbb{P} J_{\mathbb{C}^{n}, 0}^{k} \subset \mathbb{P}^{*} J_{\mathbb{C}^{n}, 0}^{k}$.

ОпрЕдЕЛЕНИЕ. Эйлерова характеристика $\chi(X)$ шилиндрического подмножества $X \subset$ $\mathbb{P} \mathscr{O}_{\mathbb{C}^{n}, 0}\left(X=\pi_{k}^{-1}(Y), Y \subset \mathbb{P} J_{\mathbb{C}^{n}, 0}^{k}\right)$ определяется как эйлерова характеристика $\chi(Y)$ множества $Y$.

ЗАмЕчАнИЕ. Полуалгебраическое подмножество конечномерного проективного пространства (например, множество $Y$ выше) может быть представлено в виде объединения конечного числа непересекающихся клеток. Эйлерова характеристика такого множества определяется как альтернированная сумма чисел клеток различных размерностей. Определенная таким образом эйлерова характеристика обладает свойством аддитивности: $\chi\left(Y_{1} \cup Y_{2}\right)=\chi\left(Y_{1}\right)+\chi\left(Y_{2}\right)-$ $\chi\left(Y_{1} \cap Y_{2}\right)$, и поэтому может рассматриваться как обобщенная (неположительная) мера на алгебре полуалгебраических подмножеств.

Пусть $\psi: \mathbb{P} \mathscr{O}_{\mathbb{C}^{n}, 0} \rightarrow A$ - функция со значениями в абелевой группе $A$.

Работа первого автора выполнена при частичной поддержке Российского фонда фундаментальных исследований (грант № 98-01-00612) и фонда INTAS (грант № 97-1644).

Работа последних двух авторов выполнена при частичной поддержке гранта DGICYT PB970471 и гранта Кастильи и Леона VA51/97. 
ОПРЕДЕЛЕНИЕ. Говорят, что функция $\psi: \mathbb{P}_{\mathbb{C}^{n}, 0} \rightarrow A$ является цилиндрической, если для любого ненулевого элемента $a \in A$ множество $\psi^{-1}(a) \subset \mathbb{P}_{\mathbb{C}^{n}, 0}$ является цилиндрическим.

ОпреДЕЛЕнИЕ. Интеграл цилиндрической функции $\psi$ по $\mathbb{P} \mathscr{O}_{\mathbb{C}^{n}, 0}$ по отношению к эйлеровой характеристике - это $\int_{\mathbb{P} \mathscr{O}_{\mathbb{C}^{n}, 0}} \psi d \chi=\sum_{a \in A, a \neq 0} \chi\left(\psi^{-1}(a)\right) \cdot a$, если эта сумма имеет смысл в $A$. Если интеграл существует (имеет смысл), то говорят, что функция $\psi$ интегрируема.

ЗАмечАниЕ. Аналогичным образом можно определить обобщенную эйлерову характеристику $[X]$ цилиндрического подмножества пространства $\mathbb{P} \mathscr{O}_{\mathbb{C}^{n}, 0}$ (или пространства $\mathscr{O}_{\mathbb{C}^{n}, 0}$ ) со значениями в кольце Гротендика комплексных полуалгебраических пространств, локализованном по классу $\mathbb{L}$ комплексной прямой, и тем самым соответствующее понятие интегрирования (см., например, [5]). Для этого можно определить $[X]$ как $[Y] \cdot \mathbb{L}^{-C_{n+k}^{k}}$.

Для особенности $C=\bigcup_{i=1}^{r} C_{i} \subset\left(\mathbb{C}^{2}, 0\right)$ плоской кривой пусть $\varphi_{i}:\left(\mathbb{C}_{i}, 0\right) \rightarrow\left(\mathbb{C}^{2}, 0\right)$ - параметризации (униформизации) компонент $C_{i}$ кривой $C$ (т.е. $\operatorname{Im} \varphi_{i}=C_{i}$ и $\varphi_{i}$ является изоморфизмом между $\mathbb{C}_{i}$ и $C_{i}$ вне начала координат). Для ростка $g \in \mathscr{O}_{\mathbb{C}^{2}, 0}$ обозначим через $v_{i}(g)$ степень начального члена в разложении в степенной ряд ростка $g \circ \varphi_{i}:\left(\mathbb{C}_{i}, 0\right) \rightarrow \mathbb{C}: g \circ \varphi_{i}\left(\tau_{i}\right)=c_{i} \cdot \tau_{i}^{v_{i}}$ + (члены более высокой степени), где $c_{i} \neq 0$. Если $g \circ \varphi_{i}(t) \equiv 0$, то полагаем $v_{i}(g)$ равным бесконечности $(\infty)$. Положим $\underline{v}(g)=\left(v_{1}(g), \ldots, v_{r}(g)\right) \in \mathbb{Z}_{\geqslant 0}^{r}, v(g)=\|\underline{v}(g)\|=v_{1}(g)+$ $\cdots+v_{r}(g)$. Пусть $\mathbb{Z}[[t]]$ (соответственно $\left.\mathbb{Z}\left[\left[t_{1}, \ldots, t_{r}\right]\right]\right)$ - группа (по отношению к сложению) формальных степенных рядов по переменной $t$ (соответственно по переменным $\left.t_{1}, \ldots, t_{r}\right)$. Для $\underline{v}=\left(v_{1}, \ldots, v_{r}\right) \in \mathbb{Z}_{\geqslant 0}^{r}$ положим $\underline{t}^{\underline{v}}=t_{1}^{v_{1}} \cdots t_{r}^{v_{r}} ;$ мы полагаем $t^{\infty}=0$.

ТеОРема 1. Для любого $\underline{v} \in \mathbb{Z}_{\geqslant 0}^{r}$ подмножество $\left\{g \in \mathbb{P} \mathscr{O}_{\mathbb{C}^{2}, 0}: \underline{v}(g)=\underline{v}\right\}$ является цилиндрическим. Поэтому функции $\underline{\underline{t}} \underline{\underline{v}}(g)$ u $t^{v(g)}$ на пространстве $\mathbb{P} \mathscr{O}_{\mathbb{C}^{2}, 0}$ со значениями в $\mathbb{Z}\left[\left[t_{1}, \ldots, t_{r}\right]\right]$ и $\mathbb{Z}[[t]]$ соответственно являются иилиндрическими.

ДокАЗАТЕЛЬСтво вытекает из того факта, что для $g \in \mathfrak{m}^{s} v_{i}(g) \geqslant s$, т.е. ряд Тейлора ростка $g \circ \varphi_{i}\left(\tau_{i}\right)$ начинается с члена степени не менее $s$. Поэтому функции $\underline{\underline{v}} \underline{v}(g)$ и $t^{v(g)}$ на $\mathbb{P} \mathscr{O}_{\mathbb{C}^{2}, 0}$ являются интегрируемыми (поскольку $\sum_{\underline{v} \in \mathbb{Z}_{\geqslant 0}^{r}} \ell(\underline{v}) \underline{\underline{v}} \underline{\underline{v}} \in \mathbb{Z}\left[\left[t_{1}, \ldots, t_{r}\right]\right]$ для любых цельх $\left.\ell(\underline{v})\right)$.

TEOPEMA 2. При $r>1 \int_{\mathbb{P} \mathscr{O}_{\mathbb{C}^{2}, 0}} \underline{t}^{\underline{v}(g)} d \chi=\Delta^{C}\left(t_{1}, \ldots, t_{r}\right) ; n p u r \geqslant 1 \int_{\mathbb{P} \mathscr{O}_{\mathbb{C}^{2}, 0}} t^{v(g)} d \chi=\zeta_{C}(t)$.

ДокАЗАТЕЛЬство следует из результатов работы [3]. Пусть $\mathbb{P} \widehat{S}_{C}$ - проективизация расширенной полугруппы кривой $C$ (см. [3]). Для $\underline{v}=\left(v_{1}, \ldots, v_{r}\right) \in \mathbb{Z}_{\geqslant 0}^{r}$ пусть $k=1+\max _{1 \leqslant i \leqslant r} v_{i}$ и пусть $Y_{\underline{v}} \subset \mathbb{P} J_{\mathbb{C}^{2}, 0}^{k}$ - множество $\left\{j^{k} g \in \mathbb{P} J_{\mathbb{C}^{2}, 0}^{k}: \underline{v}(g)=\underline{v}\right\}$ (мы указьвали, что выполнение условия $\underline{v}(g)=\underline{v}$ определяется $k$-струей $j^{k} g$ ростка $\left.g\right)$. Естественное отображение $Y_{\underline{v}} \rightarrow \mathbb{P} F_{\underline{v}}$ множества $Y_{\underline{v}}$ в слой $\mathbb{P} F_{\underline{v}}$ проективизации расширенной полугруппы $\mathbb{P} \widehat{S}_{C}$ (см. [3]) является локально тривиальным расслоением, слоем которого является комплексное линейное пространство некоторой размерности. Теперь теорема 2 вытекает из того, что $\chi\left(\mathbb{P} \widehat{S}_{C}\right)=\Delta^{C}\left(t_{1}, \ldots, t_{r}\right)$ (см. [3]).

\section{СПИСОК ЛИТЕРАТУРЫ}

[1] Eisenbud D., Neumann W. Three-Dimensional Link Theory and Invariants of Plane Curve Singularities. Princeton, NJ: Princeton Univ. Press, 1985. (Ann. of Math. Stud. V. 110.) [2] A'Campo N. // Comment. Math. Helv. 1975. V. 50. P. 233-248. [3] Гусейн-Заде С. М., Дельгадо Ф., Кампильо А. // УМН. 1999. Т. 54. № 3. С. 157-158. [4] Denef J., Loeser F. Lefschetz numbers of iterates of the monodromy and truncated arcs // Preprint math.AG/0001105. [5] Craw A. An introduction to motivic integration // Preprint math.AG/9911179.

Московский государственный университет им. М. В. Ломоносова; $\quad$ Принято редколлегией Университет г. Вальядолид, Испания

Принято редколлегией
03.10 .2000

E-mail: sabir@mccme.ru, fdelgado@agt.uva.es, campillo@cpd.uva.es 